\title{
APPROXIMATING PA
}

\author{
F. G. J. WIID
}

(Communicated by Louis J. Ratliff, Jr.)

\begin{abstract}
It is shown that the ring of continuous real-valued functions on a compact Hausdorff space of dimension $\leq 1$ is a BCS-ring. This result follows from a study of the preservation of the PA and BCS properties under certain ring-theoretic constructions.
\end{abstract}

Let $R$ be a commutative ring with identity. Then $R$ is said to be a PA-ring if each reachable system over $R$ has its poles arbitrarily assignable, and is said to be a BCS-ring, if each basic submodule of a finitely generated projective $R$-module $M$ contains a rank one projective summand of $M$ (see below for undefined terms and a more detailed discussion of these ideas). Several recent papers have been concerned with PA- and BCS-rings: e.g. $[\mathbf{1}, \mathbf{2}, \mathbf{4}, \mathbf{6}, \mathbf{7}]$. Among the more important results in this area is the following of Hautus and Sontag.

THEOREM 0. Let $X$ be a topological manifold with $C(X)$ the ring of continuous real-valued functions on $X$. Then $C(X)$ is a PA-ring if and only if $X$ has dimension $\leq 1$.

There is an intriguing formal resemblance between this theorem and the purely algebraic result that a noetherian ring is a BCS-ring (and therefore a PA-ring) provided that it has dimension $\leq 1$. (cf. $[6,7])$. Given the information that finitely generated projective modules over $C(X)$, where $X$ is a finite simplicial complex, can be represented by projective modules over a noetherian ring of dimension $\leq \operatorname{dim}(X)$ (cf. [5]), and that projective modules play a key role in the study of PA- and BCSrings, one might suspect that the relationship between the above results is more than one of formal resemblance. By adapting ideas of Swan [5] we are able to show in this paper that the Hautus-Sontag theorem is indeed a consequence of the algebraic result. Moreover, from our new vantage point it is easy to see how to obtain a generalisation of theorem 0 to the situation where $X$ is a compact Hausdorff space of dimension $\leq 1$.

This paper can also be viewed as a study of the preservation of the PA and BCS properties under certain ring theoretic constructions. One of the essential failures of PA-rings and BCS-rings is that they do not often respect ring-theoretic constructions. In Theorems 1 and 2 below, we wrest some reasonable behaviour from these recalcitrant objects.

Before we state and prove our main results we give the following definitions. A linear system over $R$ is a pair of maps $f: U \rightarrow U, g: V \rightarrow U$ between finitely

Received by the editors March 16, 1987 and, in revised form, July 3, 1987.

1980 Mathematics Subject Classification (1985 Revision). Primary 93B55. 
generated projective $R$-modules. We say that $(f, g)$ is reachable if

$$
\bigoplus_{n} V \stackrel{\oplus_{n} g}{\longrightarrow} \bigoplus_{n} U \stackrel{(f)_{i}}{\longrightarrow} U
$$

is surjective, where $n=\operatorname{rank}(U)$, and pole assignable, if, given $\left(a_{1}, \ldots, a_{n}\right) \in R^{n}$, there is a map $k: U \rightarrow V$ such that $a_{1}, \ldots, a_{n}$ are the eigenvalues of $f+g k . R$ is PA if every reachable system over $R$ is pole assignable. $R$ is PAF if every system with $U$ and $V$ free is pole assignable.

It can be shown that $R$ is PA if and only if for every reachable system $(f, g)$ over $R$ the following condition holds: $\operatorname{Im}(g)$ contains a rank one projective summand of $\operatorname{Dmn}(f)$.

A ring $R$ is a BCS-ring if every basic submodule of a finitely generated projective $R$-module $P$ contains a rank one summand of $P$. It is fairly simple to show that $R$ is a BCS-ring if and only if the following condition is satisfied: if $G$ is an $R$-matrix having unit content then there is a matrix $V$ such that $G V$ is a *-matrix (i.e. $G V$ has unit content and all $2 \times 2$ minors of $G V$ are zero) (cf. $[\mathbf{1}, \mathbf{6}]$ ).

In the sequel $f: A \rightarrow B$ is a morphism of rings such that:

(1) $B$ is a topological ring, $B *$ (the units of $B$ ) is open in $B$ and $u \rightarrow u^{-1}$ is continuous on $B *$,

(2) $f(A)$ is dense in $B$,

(3) if $f(a)$ is sufficiently near 1 in $B$, then $a \in A *$.

Swan (5) also introduces the technical condition (SBI). We shall refrain from explicating this condition-it ensures that each finitely generated projective $B$ module is extended from $A$.

There are two theorems.

THEOREM 1. Let $f: A \rightarrow B$ satisfy (1)-(3). If $A$ is a BCS-ring, then $B$ is a BCS-ring. If, in addition, $f$ also satisfies $(S B I)$, then $B$ is a PA-ring if $A$ is a PA-ring.

THEOREM 2. Let $\left\{f_{\alpha \mu}: A_{\alpha} \rightarrow A_{\mu}\right\}_{\alpha \leq \mu}$ be a directed system of rings. If each $A_{\alpha}$ is a BCS-ring (resp. a PAF-ring or a PA-ring) then so is $\longrightarrow$

PROOF OF THEOREM 1. The first assertion is proved as follows. Let $G=$ $\left(c_{i j}\right): B^{m} \rightarrow B^{n}$ be a matrix having unit content, say $1=\sum_{i, j} r_{i j} c_{i j}$ for some $r_{i j} \in B$. By (2), there exist $s_{i j}, d_{i j} \in A$ such that $f\left(s_{i j}\right)$ is arbitrarily close to $r_{i j}$ and $f\left(d_{i j}\right)$ is arbitrarily close to $c_{i j}$. Thus by (1), we can choose $s_{i j}, d_{i j}$ such that $f\left(\sum_{i, j} s_{i j} d_{i j}\right)=\sum_{i, j} f\left(s_{i j}\right) f\left(d_{i j}\right)$ is arbitrarily close to $\sum r_{i j} c_{i j}=1$. Thus we can bring $f\left(\sum_{i, j} s_{i j} d_{i j}\right)$ sufficiently close to 1 to ensure (by (3)) that $\sum_{i, j} s_{i j} d_{i j}$ is a unit. Therefore $G^{\prime}=\left(d_{i j}\right)$ has unit content. Since $A$ is a BCS ring, there is a rank one projective summand $P$ in the image of $G^{\prime}$ : that is, denoting by $\Pi$ the canonical projection, the following sequence is exact

$$
A^{m} \stackrel{\Pi \cdot G^{\prime}}{\longrightarrow} P \rightarrow 0
$$


Then the sequence

$$
B^{m} \stackrel{\left(\Pi \otimes 1_{B}\right) \cdot f\left(G^{\prime}\right)}{\longrightarrow} P \otimes B \rightarrow 0
$$

is exact and the map $\left(\Pi \otimes 1_{B}\right) \cdot G$ is sufficiently close to $\left(\Pi \otimes 1_{B}\right) \cdot f\left(G^{\prime}\right)$. Thus, by [5, Lemma 1.3], the map $\Pi \otimes 1_{B} \cdot G$ is surjective and this gives a rank one projective summand in the image of $G$. It follows that $B$ is a BCS-ring.

The proof of the second assertion is an adaptation of the above proof: one uses (SBI) to show that each reachable $B$-system can be approximated arbitrarily closely by a reachable $A$-system and then one reasons as above.

PROOF OF THEOREM 2. A proof of the BCS portion of the theorem goes as follows. Let $G \in M_{n \times m}(A)$ have unit content. Write $G=\left(a_{i j}\right)$, then there exist $b_{i j} \in A$ so that $\sum_{i, j} a_{i j} b_{i j}=1$. If $f_{\alpha}: F_{\alpha} \rightarrow \underset{\lim }{\rightarrow} F_{\alpha}$ are the universal mappings, we can find $\lambda \in I$ such that there exist $c_{i j}, d_{i j} \in A_{\lambda}$ so that $f_{\lambda}\left(c_{i j}\right)=a_{i j}$ and $f_{\lambda}\left(d_{i j}\right)=b_{i j}$. Then $f_{\lambda}\left(\left(\sum_{i, j} c_{i j} d_{i j}\right)-1\right)=0$, so there exists a $\mu \geq \lambda$ so that $f_{\lambda \mu}\left(\left(\sum_{i, j} c_{i j} d_{i j}\right)-1\right)=0$. Then $\sum_{i, j} f_{\lambda \mu}\left(c_{i j}\right) f_{\lambda \mu}\left(d_{i j}\right)=1$. Let $G_{\mu}=\left(f_{\lambda \mu}\left(c_{i j}\right)\right) \in$ $M_{n \times m}\left(A_{\mu}\right)$. Then $G_{\mu}$ has unit content, and $f_{\mu}\left(G_{\mu}\right)=G$. Since $A_{\mu}$ is BCS, there exists a matrix $B_{\mu} \in M_{m \times k}\left(A_{\mu}\right)$ such that $G_{\mu} B_{\mu}$ is a $*$-matrix. Let $B=f_{\mu}\left(B_{\mu}\right)$. Then $f_{\mu}\left(G_{\mu} B_{\mu}\right)=G B$ is a $*$-matrix over $A$.

The PAF and CAF proofs are completely analogous, but the PA assertion requires some formalism involving direct limits of categories. This is also easily adapted from [5, pp. 214-215].

These results have the following

COROLlARY. Let $X$ be a compact Hausdorff space of dimension $\leq 1$. Then $C(X)$ is a BCS-ring.

ProOF. By [3, p. 278], $X=\lim X_{\alpha}$, where $\left\{X_{\alpha}\right\}$ is an inverse system of polyhedra of dimension $\leq 1$. Then by [5, p. 215], the ring morphism $f: \lim C\left(X_{\alpha}\right) \rightarrow$ $C(X)$ satisfies (1)-(3) and so by Theorems 1 and 2 we would be finished if we knew that $C\left(X_{\alpha}\right)$ were a BCS-ring for each $\alpha$. But by [5, Theorem 6.3], for each $\alpha$ there exist a noetherian subring $B_{\alpha}$ of $C\left(X_{\alpha}\right)$ having dimension $\leq 1$. Moreover, the inclusion $B_{\alpha} \subset C\left(X_{\alpha}\right)$ satisfies (1)-(3). By [6 or 7], $B_{\alpha}$ is a BCS-ring and another application of Theorem 1 completes the proof.

ACKNOWLEDGEMENT. I hereby thank the referee for helping to make this article presentable.

\section{REFERENCES}

1. J. W. Brewer, D. Katz and W. Ullery, Pole assignability in polynomial rings, power series rings and Prüfer domains, J. Algebra 106 (1987), 265-286.

2. M. L. J. Hautus, E. D. Sontag, New results on pole shifting for parametrized families of systems, J. Pure Appl. Algebra 40 (1986), 229-244.

3. S. Mardesic, On covering dimension and inverse limits of compact spaces, Illinois J. Math. 4 (1960), 278.

4. F. Minnaar, C. G. Naude, G. Naude and F. Wiid, Pole assignability of rings of low dimension, J. Pure Appl. Algebra (to appear). 
5. R. G. Swan, Topological examples of projective modules, Trans. Amer. Math. Soc. 230 (1977), 201-234.

6. W. V. Vasconcelos and C. A. Weibel, BCS-rings (to appear).

7. F. Wiid, Noetherian rings of dimension 1 are pole assignable, J. Pure Appl. Algebra (to appear).

National Research Institute for Mathematical SCiences, CSIR, PO BoX 395, Pretoria 0001, SOUTh AFrica

Current address: Department of Mathematics, University of the Witwatersrand, No. 1, Jan Smuts Avenue, Johannesburg 2000, South Africa 